\title{
Development and Implementation of AUV for Data Acquisition and Image Enhancement
}

\author{
Manu D K, P Karthik
}

\begin{abstract}
In our proposed work, the developed Acoustic Unmanned Vehicle(AUV) moves in the direction of up/down. In general AUV is mainly used to for visual observation of the underwater environment by using a web camera. The acquired data from the AUV was preprocessed and the same image used for enhancement.. In this project, the image enhancement alogrithms has been implemented and the same has been computed for Canny Edge Detection, Hue, Luma and Saturation. From these computed results, we are able to enhance the quality of images for the conditions like low depth, low light intensity and color contrasting has been achieved using LABVIEW.
\end{abstract}

Keywords: Acoustic Unmanned Vehicle, Canny Edge Detection, LabVIEW. .

\section{INTRODUCTION}

The main aim of the project is to send a vehicle into the water and capture images in that water environment. Since images are not clear in water due to different parameters like variation in intensity of light, suspended particles, etc., we will be performing an image enhancement technique on the captured images. Acoustic Underwater Vehicle (AUV) is a ROV class tethered vehicle. They are mainly used for pipeline surveillance, deep-water exploring, as guide for deep divers, etc. They are linked to host ships where the controlling unit is present. The ROV is kept in a tether management system (TMS) during lowering through splash zone. The purpose of tether management system is used increase or decrease the length of cable, by doing this acoustic currents can be minimized. The purpose of AUV of this project is deep-water exploration.

Image handling is a technique to play out certain procedure on an image, so as to get an improved picture or to extricate some helpful data from it. It is a kind of signal processing wherein an input is an image and yield might be a image or attributes/highlights related with that image. The whole setup of AUV contains hull, where the image processing setup is kept. The movement of the AUV is stabilized with the help of BLDC motors, which are controlled using ESCs and Arduino. Altitude, speed, direction of the vehicle all are controlled through Arduino. The Image processing setup

Revised Manuscript Received on April 25, 2020.

* Correspondence Author

Manu D K*, Research Scholar, Department of ECE, K S School of Engineering and Management, Bengaluru, India. Email: manu.d.k@kssem.edu.in.

P Karthik, Professor, Department of ECE, K S School of Engineering and Management, Bengaluru, Karnataka, India. Email: karthik.p@kssem.edu.in.

(C) The Authors. Published by Blue Eyes Intelligence Engineering and Sciences Publication (BEIESP). This is an open access article under the CC BY-NC-ND license (http://creativecommons.org/licenses/by-nc-nd/4.0/) consists of a USB Camera, myRIO and Laptop. The myRIO kit contains

the Image processing algorithm. The processed images are displayed on the laptop. There are models that perform the same operations, but our aim is to make it cost efficient.

\section{LITERATURE SURVEY}

Remotely Operated Vehicle (ROV) is an acoustic umanned vehicle (AUV) used for performing underwater tasks to reduce the human diver in these kind of risky job. X4-ROV is used to monitor and observe the object in a micro level. These ROVs plays major role in visual observation of underwater environment with help of web camera. The planned vehicle structure points towards the likelihood and mobility in disposition movements of move, pitch, yaw and the translational forward/switch/sidelong movement [1]. As far as the vulnerabilities incited by wave unsettling influences when a submerged vehicle is worked from the outside of water, a powerful model dependent on CFD is worked to break down 6DOF situating consolidating with the curiosity Model-Converted Remotely Operated Vehicle (MC-ROV) of sea designing [2]. Fish bounty estimation with the guide of visual investigation has drawn expanding consideration dependent on the submerged recordings from a remotely worked vehicle (ROV) [3]. MBARI has been leading remotely worked vehicle (ROV)- based video overviews of the upper 1000 meters of the water segment in Monterey Bay, California for more than 23 years [4]. Operator framework is a methodology to improve the submerged control. The ordinary control is for the most part robot arm-based arrangement that has solitary focuses. Then again, the operator framework is an armless control that the specialist vehicle functions as the end-effector [5]. ROV Bus, a correspondence structure custom-made to the custom ROV improvement that empowers fitting and-play interconnection of different segment instruments by means of a brought together interchanges transport [6].

Image upgrade process comprise of an assortment of methods that look to improve the visual appearance of a image, or to change over the image to a structure more qualified for examination by a human visual framework or for machine discernment. In numerous useful circumstances, the brightening of a scene is changing with reality. It isn't strange to have images having brilliantly lit and shadowed bits in a similar scene requesting data extraction from the whole image [7]. Image upgrade in Geographic object based Image Analysis (GEOBIA) is a promising system for picture investigation, where images are first portioned into image fragments (or items) and afterward dissected dependent on shape, surface, setting and phantom highlights [8]. 
Acoustic images acquired utilizing a $25 \mathrm{MHz}$ Bragg diffraction imaging framework were digitized into 256x256 picture components (pixels) with 8-piece quantization. Advanced preparing methods in the spatial area, the recurrence space, and the sequency (Walsh changes) area were utilized to improve picture appearance and to dismiss undesirable commotion [9]. In sound imaging, the situation of a hazy edge might be dictated by looking at where the power has dropped to one-fourth of that a long way from the edge in the brilliant area, the exactness with which this point might be found relying upon the slant of the profile [10]. Film-grain commotion seriously restricts the opportunities for improving debased photographic pictures. Since the grain commotion increments monotonically with the photo's mean or predisposition transmittance, poor sign to-clamor proportions result at whatever point the emulsion gets a low-differentiate, elevated level presentation [11].

Low-light picture upgrade technique utilizing the variational-advancement based Retinex calculation. The proposed improvement technique first gauges the underlying light and uses its gamma revised variant to compel the enlightenment part [12]. Camera-shake is unavoidable during the presentation time is sufficiently long to have attractive SNR. This work presents an video catching technique that improves picture quality in low-light conditions by intertwining noticeable band (400 $700 \mathrm{~nm})$ picture and wide-band (400 $1000 \mathrm{~nm}$ ) picture. Wide-band picture has lower clamor, more extravagant detail and a monotonic shading. Unmistakable band picture has higher clamor, more unfortunate detail and a trichromatic shading. We portray how the benefits of these pictures are abused to make new picture that is of higher caliber. Trials exhibit that the proposed technique fundamentally improves nature of pictures under low light conditions [13]. A non-linear upgrade work has been planned to dependent on the neighborhood scattering of the wavelet coefficients displayed as a bi-variate Cauchy appropriation. Inside the equivalent measurable structure, a concurrent clamor decrease in the picture is performed by methods for a shrinkage work, accordingly forestalling commotion intensification [14]. A hearty difference improvement calculation for loud low-light pictures, known as Denoising enhancement completion (DEC), has been proposed here [15]. [16] In this work, an extraordinary failure light multi-outline combination calculation to get a brilliant and clear shot significantly under dull conditions. To this end, utilizing numerous short-introduction pictures and one appropriate presentation foggy picture as an info, another progressive square savvy transient clamor separating is finished. Up until this point, by methods for the investigation of low-light picture from DMSP/OLS, numerous items can be gotten. These items are utilized in numerous zones, for example, urban degrees assessment, populace studies and evening time low cloud and mist identification [17].

\section{DESIGN AND IMPLEMNETATION}

The proposed system consists of two units:

- Image Processing Unit

- AUV unit

\section{A. Image Processing Unit}

The image-processing section contains a camera where the images are captured. The captured images are given to
myRIO as shown Figure 1. For image processing in LabVIEW, we use two modules namely, Vision Development Module (VDM) and Vision Acquisition Module (VAM). The image-processing algorithm so developed uses the above-mentioned module and will be dumped to myRIO, which will act as a standalone system, and it can monitor through tether/Wi-Fi. The final enhanced image will be displayed on the screen.

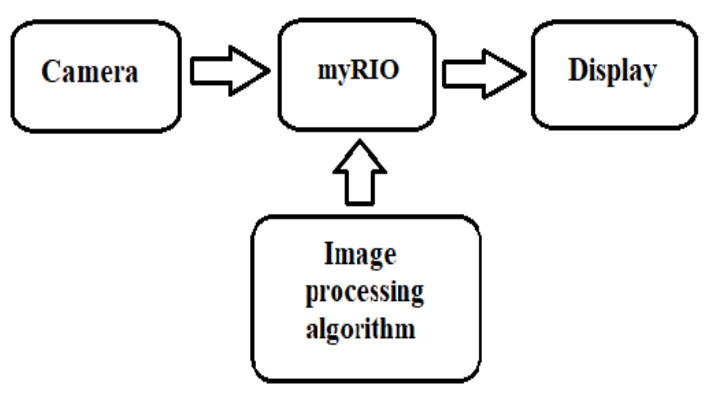

Figure 1 Image Processing Unit

\section{B. Auv Unit}

AUV consists of two subsystems. Where in mechanical system, it has a hull part and BLDC motor. The whole Image processing setup is placed inside the hull. BLDC motor, the essential rule of activity is like a dc engine. A BLDC has a rotor with lasting magnets and a stator with windings. It is basically a dc engine turned back to front. The brushes and commutators have been disposed of and the windings are associated with the gadgets control circuit. By using this, the complete movement of AUV is achieved by buoyancy i.e., aim towards portability in height movements of pitch and yaw and interpretation movement, for example, forward, turn around and lateral. Figure 3 shows the proposed AUV model. Electronic speed control (ESC) is an electronic circuit with the reason to differ a servomotor's speed, its bearing and perhaps at the same time to go about as a unique brake. ESCs are regularly utilized on engines basically giving an electronically produced three-stage electric force low voltage wellspring of vitality for the engine. It likewise permits a lot of smoother and increasingly exact variety of engine speed in an unquestionably more proficient way than the mechanical kind with a resistive curl and moving arm the once in like manner use.

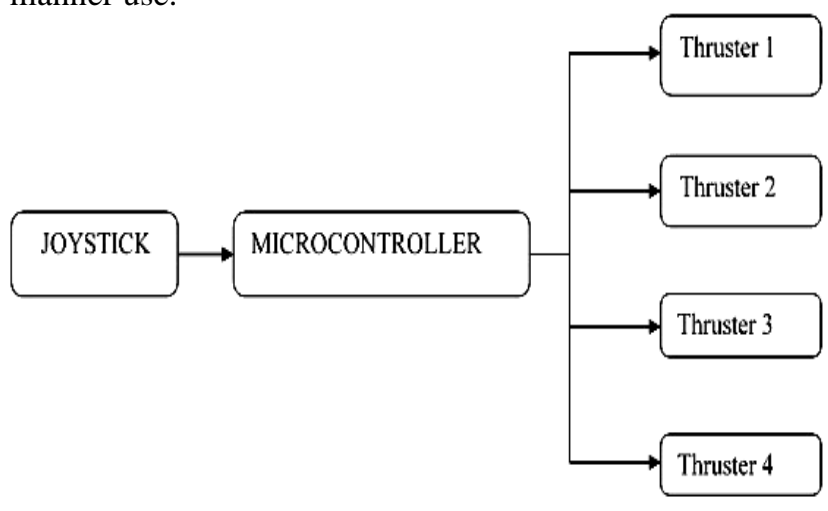

Figure 2 AUV Unit

Published By:

Blue Eyes Intelligence Engineering \& Sciences Publication 
A communication framework is an assortment of individual correspondence systems, transmission framework normally fit for interconnection and between activity to shape a coordinated entirety. The segments of a communication framework fill a typical need, are actually perfect, utilize regular strategies, react to controls, and work in association. A communication framework is a useful unit or an operational get together.

The both ESC and communication system is controlled by a Microcontroller as shown in Figure 2, where the Arduino mega is the controller. By using Arduino, the power supply for ESC is provided and the program to control the motor speed is dumped.

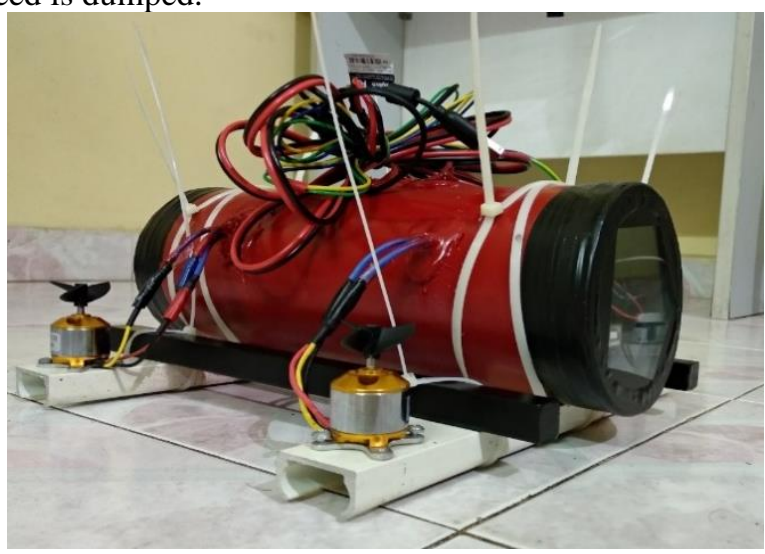

Figure 3 AUV Model

\section{Thrust Requirement For Auv}

It is required to decide push prerequisite for the vehicle with the goal that a suitable engine estimating are met. A force of drag affects the thrust of AUV which is calculated using equation (1). At the point when a vehicle is traveling through the liquid, the quick encompassing liquid is quickened alongside the body accordingly influencing the elements of the vehicle. Such power is known as included mass where it is relying upon the state of the vehicle, and liquid thickness. From,

$$
F=M a
$$

In addition, the characteristics of added mass, the total mass matrix $M$ of the body is given by equation (2):

$$
M=M_{f} I+M_{F}
$$

where $M_{b}=$ mass of vehicle,

$M_{F}=$ added mass,

$I=3 \times 3$ identity matrix.

For a cylindrical body $M_{F}$ in water is shown in equation (3),

$$
M_{f}=\rho \pi R^{2} L
$$

where $\rho$ =density of fluid, $R=$ radius, $L=$ vehicle length. By using specification of cylindrical body as listed in Table 1 , and using equation (4) we can calculate required thrust for $0.5 \mathrm{~m} / \mathrm{s}$ acceleration using the formula

$$
F=\left(M_{b}+M_{f}\right) a
$$

By assuming, $\rho=1000 \mathrm{~kg} / \mathrm{m}^{3}$ gives $M_{f}=3.123 \mathrm{~kg}, M_{b}=3.7$ $\mathrm{kg}$ and $F=3.41 \mathrm{~N}$
Table 1 Specifications of Cylindrical Body

\begin{tabular}{|c|c|c|}
\hline Radius & Length & Total Mass \\
\hline $5.6 \mathrm{~cm}$ & $31.7 \mathrm{~cm}$ & $3.7 \mathrm{~kg}$ \\
\hline
\end{tabular}

\section{RESULTS AND DISCUSSIONS}

The main aim of our project was to design and implement an Acoustic Underwater Vehicle along with the data acquisition and image enhancement. The use of this proposed model is that to capture the different underwater images and enhance them using Lab VIEW tool even for the low light images.

The designing process involves the use of LabVIEW tool in which Vision Acquisition and Vision Assistant are used. Vision Acquisition is used for the acquiring of the images from the system or from the source and Vision Assistant is used to enhance the properties of the specific image based on the different set of algorithms. Our project involves various algorithms for image enhancement such as canny edge detection, Hue, Luma and Saturation. Images captured vary from low light to high intensity bright images.

\section{A. Canny Edge Detection}

Canny edge recognition is a procedure to remove helpful basic data from various vision objects and drastically lessen the measure of information to be handled. It has been generally applied in different PC vision frameworks. Watchful has discovered that the prerequisites for the use of edge recognition on assorted vision frameworks are moderately comparable. In this way, an edge location answer for address these prerequisites can be actualized in a wide scope of circumstances. First the image will be acquired from the system using Vision Acquisition and that is being sent to the IMAQ DAQ to store and read the image and that is sent to the Canny edge detection block where the operation will be performed and finally the processed image is sent to the output for the display. The Canny edge detection block diagram is as shown in figure 4.

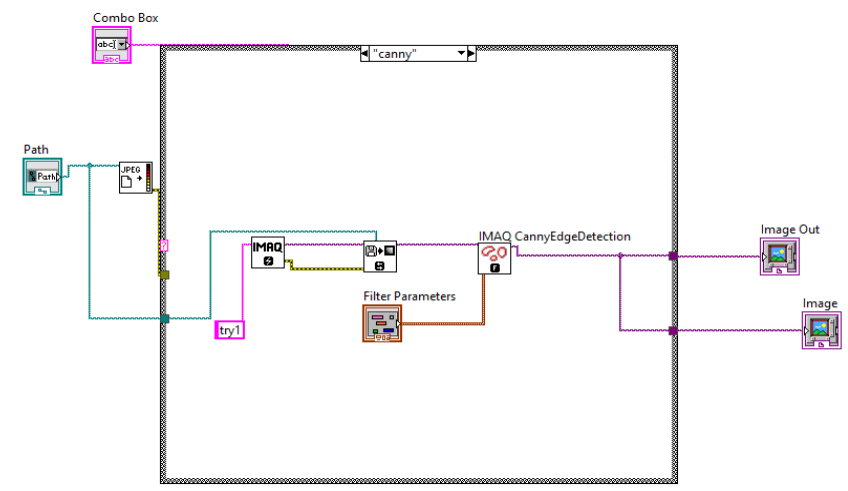

Figure 4 Block Diagram of Canny Edge Detection

The Canny edge algorithm is an edge identification administrator that utilizes a multi-arrange calculation to distinguish a wide scope of edges in images as shown in Figure 5.

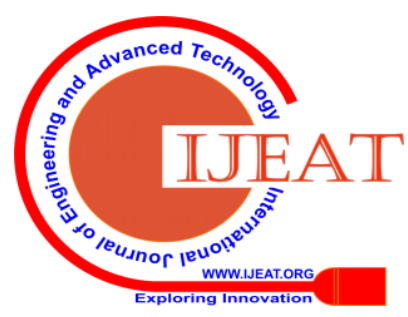




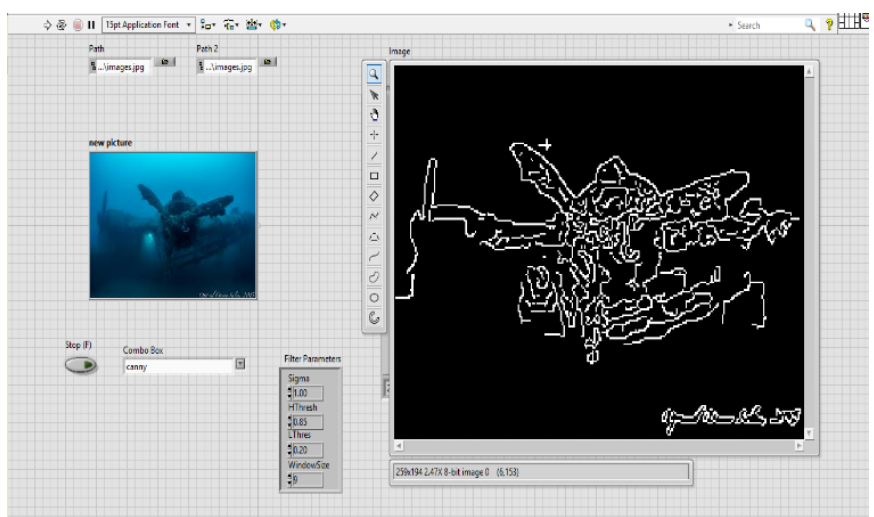

Figure 5 Processed Output Canny Edge Detector

\section{B. Luma}

Luma speaks to the splendor in a picture (the "highly contrasting" or colorless bit of the picture). Luma is ordinarily matched with chrominance. Luma speaks to the colorless picture, while the chroma segments speak to the shading data. Luma is the weighted total of gamma-packed $\mathrm{R}^{\prime} \mathrm{G}^{\prime} \mathrm{B}^{\prime}$ segments of a shading video - the prime images ' signify gamma pressure. Relative luminance is shaped as a weighted entirety of straight RGB parts, not gamma-compacted ones. All things considered, luma is incorrectly called luminance. SMPTE EG 28 prescribes the image $\mathrm{Y}^{\prime}$ to signify luma and the image $\mathrm{Y}$ to indicate relative luminance.

The block diagram for Luma is as shown in Figure 6 and the processed output image is as shown in Figure 7.

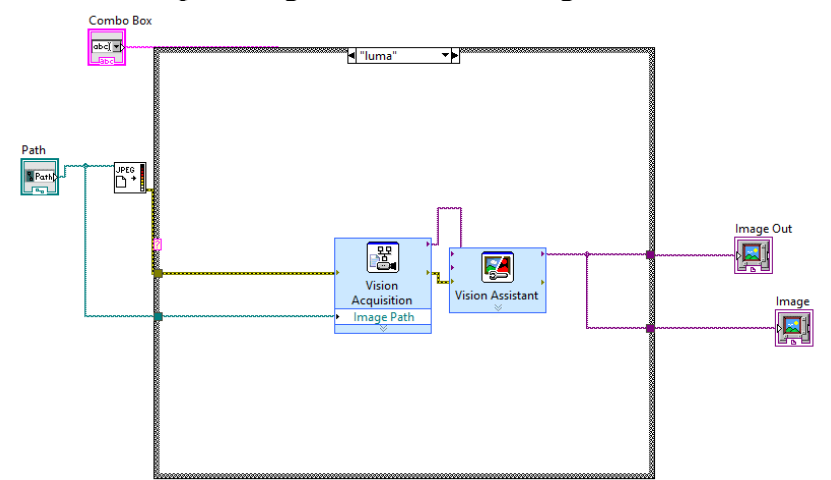

Figure 6 Block Diagram of LUMA

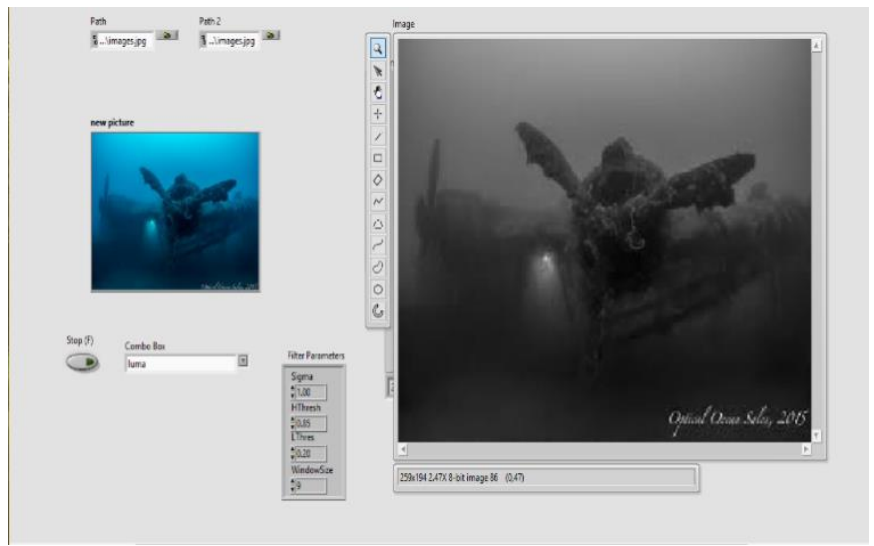

Figure 7 Processed Output of LUMA

\section{HUE}

Hue is one of the fundamental properties (called shading appearance parameters) of a shading, characterized in fact (in the CIECAM01 model), as "how much a boost can be depicted as like or not quite the same as improvements that are portrayed as red, green, blue and yellow. Tint can ordinarily be spoken to quantitatively by a solitary number, frequently comparing to a precise situation around a focal or impartial point or hub on a shading space facilitate graph, (for example, a chromaticity outline) or shading wheel, or by its prevailing frequency or that of its correlative shading. The other shading appearance parameters are colourfulness, immersion (otherwise called force or Chroma, gentility, and splendor).

The block diagram of Hue is as shown in in figure 8 and figure 9 shows the processed output image.

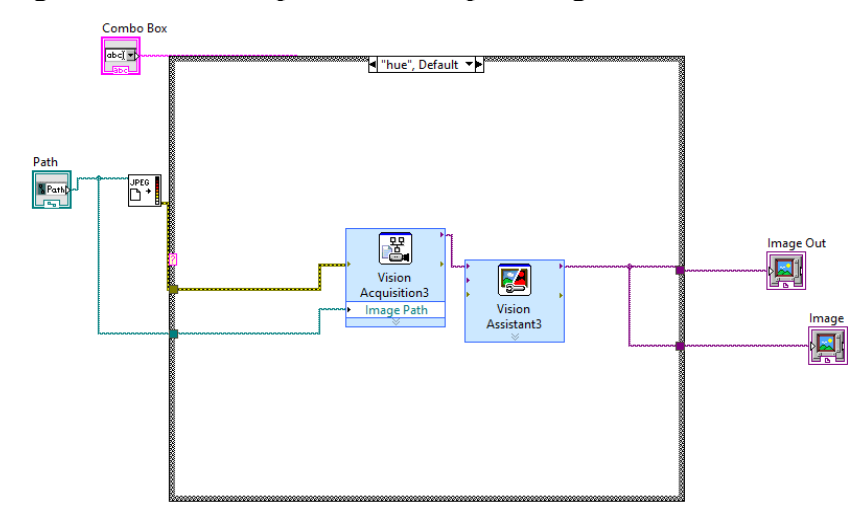

Figure 8 Block Diagram of HUE

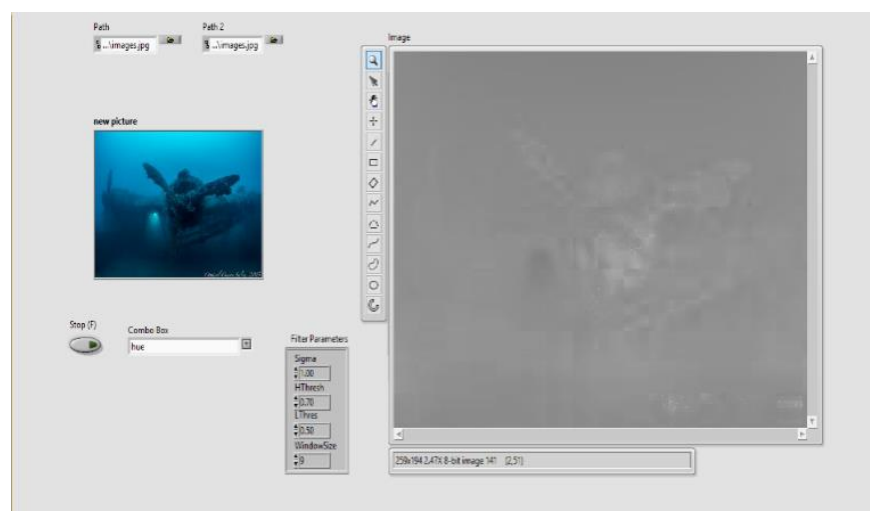

Figure 9 Processed Output of HUE

\section{Saturation}

Saturation is an attribute of the perceived color relating to chromatic intensity. Saturation is the "vividness of a region decided in relation to its splendor", which as a result is the apparent opportunity from whitishness of the light originating from the region. A note going with this definition in actuality demonstrates that an article with a given ghostly reflectance displays roughly steady immersion for all degrees of brightening, except if the splendor is high. Since the chroma and daintiness of an article are its beauty and splendor decided with respect to something very similar ("the brilliance of a comparably lit up region that seems white or profoundly transmitting"), the immersion of the light originating from that item is as a result the chroma of the item decided in relation to its gentility. The immersion of a shading is dictated by a blend of light power and the amount it is dispersed over the range of various frequencies.

The figure 10 shows the block diagram of saturation algorithm devolped in LABVIEW. The figure 11 shows the processed output of the saturation. 


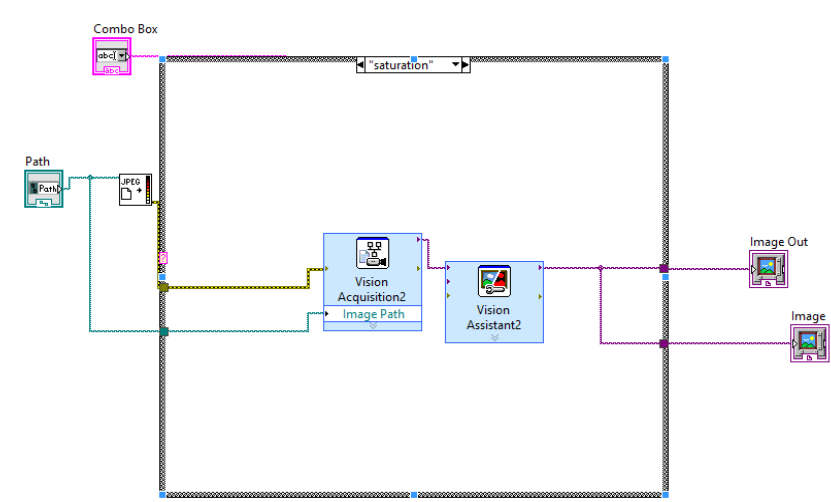

Figure 10 Saturation algorithm in LABVIEW

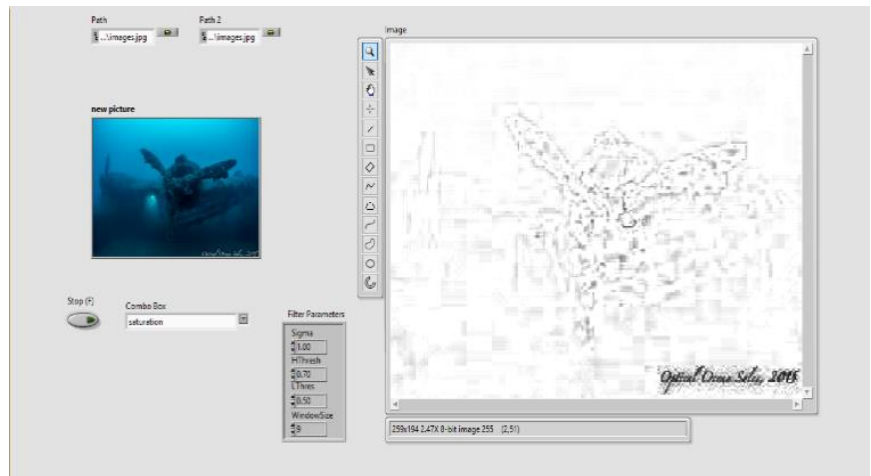

Figure 11 Processed Output of Saturation

Further the image processing was designed for continuous video acquired through webcam placed in the hull. The output of algorithms for image and video were similar. The front panel for video acquisition and processing is as shown in Figure 12.

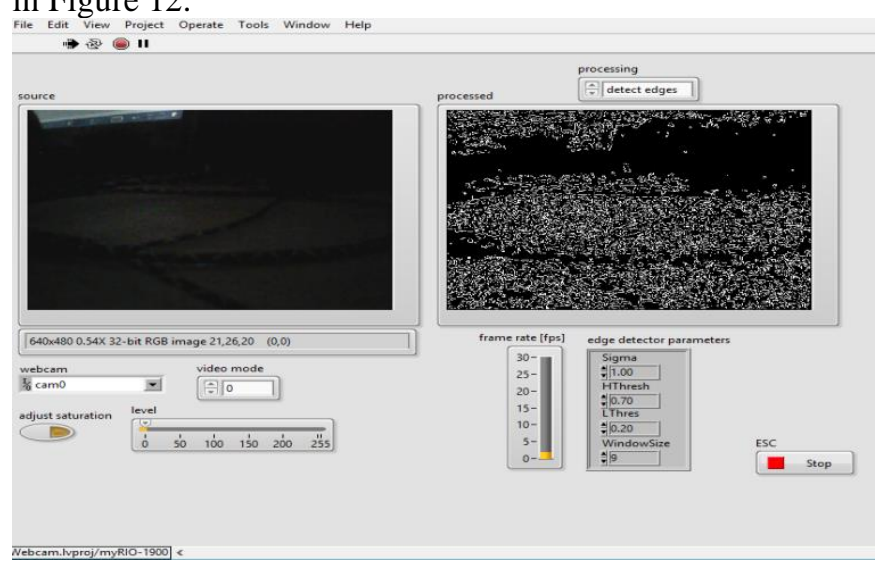

Figure 12 Front Panel of Live Video Acquisition

\section{CONCLUSION}

A detailed study of different underwater image capturing techniques and types of underwater vehicles was studied to identify the various technologies involved, also summarize the complexity involved in the existing concepts and AUVs by referring the specific journals and conference papers. The developed model, AUV, moves in underwater environment with the help of thrusters. Then we have mounted a camera to this developed AUV to capture the images in the water. Vehicle has been designed to ensure that it is of optimal weight, has sufficient thrust and is water tight. For the image processing part, we have used the LabVIEW tool, which provides ample number of image processing techniques for the images. Pictures have been caught at various levels and the equivalent has been given to improve the nature of the picture by expanding the complexity for the low light pictures. The captured images were processed using various image processing algorithms such as Canny edge detection, Hue , Luma and Saturation and the desired effectiveness of these algorithms were demonstrated. Hence, low light images are enhanced using the above-mentioned techniques. Live video stream using webcam is also accomplished and for that various image processing algorithms has also been accomplished.

\section{ACKNOWLEDGMENT}

We would like to thank the Naval Research Board for funding this project. We gratefully thank the Visvesvaraya Technological University, Jnana Sangama, Belagavi for the financial support extended to this research work. Also, we are thankful to our colleagues Mr.Gopalkrishnamurthy C R, Mr. Ravikiran B A and Head of the Department ECE, KSSEM, Bengaluru for useful suggestions and comments

\section{REFERENCES}

1. Z. M. Zain, M. M. Noh, K. A. Ab Rahim and N. Harun, "Design and development of an X4-ROV,” 2016 IEEE International Conference on Underwater system technology: Theory and Applications(USYS), Penang, PP-207-211, 2016.

2. Z. Qingiun, L. Song, L. Huiting, Z. Ming and D. Xiaoqiang, "Research on dynamic positioning of Model-converted ROV Anti-Waves based on Micro Inertial navigation sensors," 2016 10th International conference on sensing technology (ICST), Nanjing, PP- 1-6, 2016.

3. G. Wang, J. N. Hwang, K. Williams and G. Cutter, "Closed loop tracking by detection for ROV based multiple fish tracking," 2016 ICPR 2nd workshop on Computer Vision for analysis of underwater imagery(CVAUI), Cancun, PP- 7-12, 2016.

4. K. R. Reisenbichler, "Automating MBARI's midwater time-series video surveys: the Transition from ROV to AUV," OCEANS 2016 MTS/IEEE Monterey, Monterey,CA, PP- 1-9, 2016.

5. J. Kim and S. C. Yu, "Convolutional Neural network-based real-time ROV detection using forward-looking Sonar image," 2016 IEEE/OES Autonomous underwater vehicle(AUV), Tokyo, PP- 396-400, 2016.

6. M. Cook, G. Cook, E. Celkis and T. Crandle, "ROV Bus: A modular electronics backbone for flexible ROV capability integration," OCEANS 2016 MTS/IEEE Moneterey, Moneterey,CA, PP- 1-4, 2016.

7. N. Mishra, S. P. Kumar, R. Chandrakanth and R. Ramachandran, "Image enhancement using Logarithmic image processing model," IETE Journal of Research, Volume 46, Issue 5, 2000.

8. P. R. Marpu, M. Neubert, H. Herold and I. Niemeyer, "Enhancement evaluation of Image segmentation results," Journal of Spatial science, Volume 55, Issue 1, 2010.

9. "Digital image enhancement techniques applied to acoustic images," Ultrasonics Synopsium, 1973.

10. C. J. R Sheppard and D. K. Hamilton, "Edge enhancement by Defocusing of Confocal images," OPTICA ACTA, Volume 31, Issue 6, PP - 723-727, 1984.

11. D. G. Falcorner, "Image enhancement and Film-Grain noise," OPTICA ACTA, Volume 17, PP-693-705, 1970.

12. S. Park, B. Moon, S. Ko, S. Yu and J. Paik, "Low light image restoration using bright channel prior-based variational retinex model," EURASIP Journal on Image and video processing, Volume-2017, Issue 1, 2017.

13. Y. Yoo, w. Choe, J. Kwon, S. Park, S. Lee and C. Y. Kim, "Low light imaging method with visible band and wide band image pair," 2009 16th IEEE International Conference on Image processing (ICIP), Cairo, PP- 2773-2776, 2009.

14. A. Loza, D. Bull and A. Achim, "Automatic contrast enhancement of low light images based on local statistics of wavelet coefficients," 2010 IEEE International conference on image processing, Hong Kong, PP3553-3556, 2010. 
15. J. Lim, J. H. Kim, J. Y. Sim and C. S. Kim, "Robust contrast enhancement of nosiy low light images: Denoisy-enhancement-completion,” 2015 IEEE International conference on image processing (ICIP), Quebec City,QC, PP4131-4135, 2015.

16. Y. S. Moon, S. H. Lee, Y. M. Tai and J. Cho, "A fast low light multi-image fusion with online image restoration," 2013 IEEE International conference on consumer electronics, Las Vegas,NV, PP346-347, 2013.

17. Y. Huang, S. Ma, X. Zhou and K. Wang, "A new light detection algorithm for low light images from DMSP/OLS," 2012 2nd International conference on remote sensing, Environment and Transportation Engineering, Nanjing,Jiangsu,China, PP- 1-4, 2012.

\section{AUTHORS PROFILE}

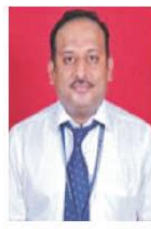

Manu D K is currently working as an Associate Professor in the Department of Electronics and Communication Engineering at K S School of Engineering and Management, Bengaluru, Karnataka, India. He has completed graduation (B.E) and Post-Graduation (M.Tech) from Visveswaraya Technological University (VTU). He is pursuing his Ph.D. in VTU. He has a passion for teaching and guided several students in UG and PG projects. He has published several papers in National and International Journals. He is also a member of the Institution of Engineers India (IEI) and ISTE.

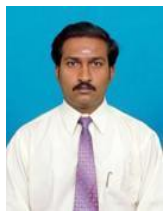

Dr.Karthik $\mathbf{P}$ is currently working as a Professor in the Department of Electronics and Communication Engineering at $\mathrm{K} S \mathrm{~S}$ School of Engineering and Management, Bengaluru, Karnataka, India. He received his B.E degree in 2004 and M.E degree in 2006. He received his Ph.D. degree in the year 2013. He has a passion for teaching and guided several students in UG and PG projects. He is currently guiding 6 research scholars in various domains. He has published several papers in National and International Journals. He is also a member of the Institution of Engineers India (IEI) and ISTE.

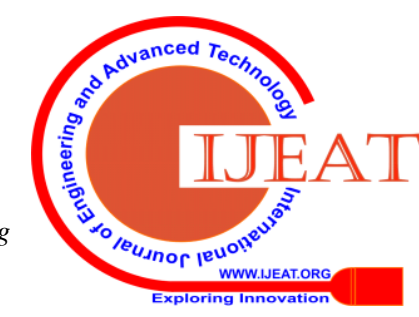

